TRANSACTIONS OF THE

AMERICAN MATHEMATICAL SOCIETY

Volume 354, Number 12, Pages 4909-4920

S 0002-9947(02)03089-1

Article electronically published on August 1, 2002

\title{
SPHERICAL NILPOTENT ORBITS AND THE KOSTANT-SEKIGUCHI CORRESPONDENCE
}

\author{
DONALD R. KING
}

\begin{abstract}
Let $G$ be a connected, linear semisimple Lie group with Lie algebra $\mathfrak{g}$, and let $K_{\mathbf{C}} \rightarrow \operatorname{Aut}\left(\mathfrak{p}_{\mathbf{C}}\right)$ be the complexified isotropy representation at the identity coset of the corresponding symmetric space. The Kostant-Sekiguchi correspondence is a bijection between the nilpotent $K_{\mathbf{C}}$-orbits in $\mathfrak{p}_{\mathbf{C}}$ and the nilpotent $G$-orbits in $\mathfrak{g}$. We show that this correspondence associates each spherical nilpotent $K_{\mathrm{C}}$-orbit to a nilpotent $G$-orbit that is multiplicity free as a Hamiltonian $K$-space. The converse also holds.
\end{abstract}

\section{INTRODUCTION}

Let $\mathbf{L}$ be a complex, connected reductive Lie group, and let $X$ be an algebraic variety on which $\mathbf{L}$ acts. $X$ is a spherical variety for $\mathbf{L}$ if there is a Borel subgroup $B=B(\mathbf{L})$ of $\mathbf{L}$ that has a dense orbit in $X$. The spherical varieties for $\mathbf{L}$ have a number of special properties from the standpoint of the representation theory of $\mathbf{L}$. For example, each irreducible finite-dimensional representation of $\mathbf{L}$ occurs at most once in the semisimple $\mathbf{L}$-module $R[X]$, the ring of regular functions on $X . R[X]$ is said to be multiplicity free as an $\mathbf{L}$-module.

Some of the most important spherical varieties for reductive groups consist of nilpotent elements that are associated to related symmetric spaces. To describe these varieties we must introduce some additional notation. Assume that $\mathfrak{g}$ is a real semisimple Lie algebra with Cartan decomposition $\mathfrak{g}=\mathfrak{k} \oplus \mathfrak{p} . \quad \theta$ is the associated Cartan involution. Let $\mathfrak{g}_{\mathrm{C}}, \mathfrak{k}_{\mathrm{C}}$ and $\mathfrak{p}_{\mathrm{C}}$ denote the complexifications of $\mathfrak{g}, \mathfrak{k}$ and $\mathfrak{p}$ respectively. $\theta$ extends to a complex linear involution on $\mathfrak{g}_{\mathrm{C}}$. Let $\sigma$ denote conjugation on $\mathfrak{g}_{\mathrm{C}}$ relative to the real form $\mathfrak{g}$. $G$ is a connected, linear semisimple Lie group with Lie algebra $\mathfrak{g} . G_{\mathrm{C}}$ is the complexification of $G . K$ and $K_{\mathrm{C}}$ are the connected subgroups of $G_{\mathrm{C}}$ with the Lie algebras $\mathfrak{k}$ and $\mathfrak{k}_{\mathrm{C}}$, respectively.

$G_{\mathrm{C}}$ has finitely many nilpotent orbits in $\mathfrak{g}_{\mathrm{C}}$. Since the pioneering work of Dynkin in the 1940s, these orbits have been studied intensively by many researchers. If $G_{\mathrm{C}}$ is simple, the spherical nilpotent orbits in $\mathfrak{g}_{\mathrm{C}}$ have been determined by Panyushev [10] and McGovern [7. Spherical orbits have become significant in the study of the completely prime primitive ideals in the enveloping algebra of $\mathfrak{g}_{\mathrm{C}}$. For example, McGovern has shown how to associate Dixmier algebras to spherical nilpotent orbits (and their covers) [7. The Dixmier algebra associated to a spherical orbit has a nice structure owing to the fact that the coordinate ring of the orbit is multiplicity-free.

$K_{\mathrm{C}}$ has finitely many nilpotent orbits in $\mathfrak{p}_{\mathrm{C}}$. These orbits have also been studied by many authors, notably Kostant and Rallis in their fundamental paper [6]. A

Received by the editors February 7, 2001 and, in revised form, April 16, 2002.

2000 Mathematics Subject Classification. Primary 22E46; Secondary 14R20, 53D20.

(C)2002 American Mathematical Society 
minimal nonzero nilpotent orbit of $K_{\mathbf{C}}$ in $\mathfrak{p}_{\mathbf{C}}$ is spherical for $K_{\mathbf{C}}$. If $\mathfrak{g}$ is the split real form of $E_{8}$, the 64-dimensional nilpotent $K_{\mathbf{C}^{-}}$-orbit in $\mathfrak{p}_{\mathbf{C}}$ is another example of a spherical orbit. In each of these examples, the "orbit method" (as defined in [14]) attaches very interesting unitary representations to the universal cover of $G$. See [15] and [1]. These works illustrate the value of a deeper understanding of the spherical nilpotent orbits of $K_{\mathbf{C}}$ in $\mathfrak{p}_{\mathbf{C}}$.

The Kostant-Sekiguchi correspondence associates to each nilpotent $K_{\mathbf{C}}$-orbit $\mathcal{O}$, a unique nilpotent $G$-orbit $\Omega=\Omega_{\mathcal{O}}$ in $\mathfrak{g}$. (One feature of this correspondence is that both $\mathcal{O}$ and its image $\Omega$ are contained in the same $G_{\mathbf{C}}$-orbit in $\mathfrak{g}_{\mathbf{C}}$.) Because sphericality is so special, it is natural to explore the properties of the image of a spherical nilpotent orbit under the Kostant-Sekiguchi correspondence. The answer is very neat and precise. It is given by Theorem 6.4.

Theorem. $\mathcal{O}$ is spherical for $K_{\mathrm{C}}$ if and only if $\Omega_{\mathcal{O}}$ is multiplicity free as a Hamiltonian $K$-space.

The multiplicity-free spaces obtained from Theorem 6.4 are sometimes closely related to other more well-known examples such as those studied by Guillemin and Sternberg in [3]. For example, if $\mathfrak{g}=s l(2 n, \mathbf{C})$, then the Kostant-Sekiguchi correspondence identifies each nilpotent $K_{\mathbf{C}}$-orbit $\mathcal{O}$ with itself, that is, $\mathcal{O}=\Omega_{\mathcal{O}}$. The orbit $\mathcal{O}$ corresponding to the partition $2^{n}$ (i.e., all parts of the partition have size 2) is spherical for $S L(2 n, \mathbf{C})$ and is diffeomorphic to a dense open set of the cotangent bundle of the compact symmetric space $S U(2 n) / S(U(n) \times U(n))$. Guillemin and Sternberg showed that this cotangent bundle is multiplicity free for $K=S U(2 n)$.

\section{Kostant-Sekiguchi CORRESPOndence}

An ordered triple $\left\{Z_{1}, Z_{2}, Z_{3}\right\}$ of elements in $\mathfrak{g}_{\mathbf{C}}$ (or $\left.\mathfrak{g}\right)$ is said to be an $s l(2)$-triple if the following commutation relations are satisfied:

$$
\left[Z_{1}, Z_{2}\right]=2 Z_{2},\left[Z_{1}, Z_{3}\right]=-2 Z_{3} \text {, and }\left[Z_{2}, Z_{3}\right]=Z_{1} \text {. }
$$

Two $s l(2)$-triples $\left\{Z_{1}, Z_{2}, Z_{3}\right\}$ and $\left\{Z_{1}^{\prime}, Z_{2}^{\prime}, Z_{3}^{\prime}\right\}$ are said to be conjugate under a subgroup $\mathbf{W}$ of $G_{\mathbf{C}}$ if there exists an element $w \in \mathbf{W}$ such that $Z_{i}=w \cdot Z_{i}^{\prime}$ for $i=1,2,3$. ("." denotes the adjoint action.)

Definition 2.1. Let $\mathcal{N}[\mathfrak{g}], \mathcal{N}\left[\mathfrak{g}_{\mathrm{C}}\right]$, and $\mathcal{N}\left[\mathfrak{p}_{\mathrm{C}}\right]$ denote the set of nilpotent elements of $\mathfrak{g}, \mathfrak{g}_{\mathrm{C}}$, and $\mathfrak{p}_{\mathrm{C}}$ respectively. $\mathcal{N}[\mathfrak{g}] / G, \mathcal{N}\left[\mathfrak{g}_{\mathrm{C}}\right] / G_{\mathrm{C}}$, and $\mathcal{N}\left[\mathfrak{p}_{\mathrm{C}}\right] / K_{\mathrm{C}}$ will denote the orbits (conjugacy classes) in $\mathcal{N}[\mathfrak{g}], \mathcal{N}\left[\mathfrak{g}_{\mathbf{C}}\right]$, and $\mathcal{N}\left[\mathfrak{p}_{\mathbf{C}}\right]$ under $G, G_{\mathbf{C}}$, and $K_{\mathbf{C}}$ respectively.

Using the Jacobson-Morosov Theorem, one can prove

Theorem 2.2. There is a bijection between each of the following sets:

(1) $G$ conjugacy classes of $\operatorname{sl}(2)$-triples of $\mathfrak{g}$;

(2) $\mathcal{N}[\mathfrak{g}] / G$.

The $s l(2)$-triple $\left\{Z_{1}, Z_{2}, Z_{3}\right\}$ is said to be normal if $Z_{1} \in \mathfrak{k}_{\mathbf{C}}$, and $Z_{2}, Z_{3} \in \mathfrak{p}_{\mathbf{C}}$.

Normal $\operatorname{sl}(2)$-triples are at the heart of the Kostant-Rallis description of $\mathcal{N}\left[\mathfrak{p}_{\mathbf{C}}\right]$ $/ K_{\mathbf{C}}$, which gives an analog of Theorem 2.2 .

Theorem 2.3. There is a bijection between each of the following sets:

(1) $K_{\mathbf{C}}$ conjugacy classes of normal sl(2)-triples of $\mathfrak{g}_{\mathbf{C}}$;

(2) $\mathcal{N}\left[\mathfrak{p}_{\mathbf{C}}\right] / K_{\mathbf{C}}$. 
In order to define the Kostant-Sekiguchi correspondence, we need to define two notable classes of $s l(2)$-triples.

Definition 2.4 (Kostant-Sekiguchi triples). An $s l(2)$-triple $\{H, E, F\}$ in $\mathfrak{g}$ is said to be a KS-triple in $\mathfrak{g}$ if $\theta(E)=-F$, and hence $\theta(H)=-H$. A normal $s l(2)$-triple $\{x, e, f\}$ in $\mathfrak{g}_{\mathrm{C}}$ is said to be a KS-triple in $\mathfrak{g}_{\mathrm{C}}$ if $f=\sigma(e)$.

Sekiguchi established the following connection between KS-triples and $s l(2)-$ triples.

Theorem 2.5 ([12]). (1) Every sl(2)-triple $\left\{H^{\prime}, E^{\prime}, F^{\prime}\right\}$ in $\mathfrak{g}$ is conjugate under $G$ to a KS-triple in $\mathfrak{g}$. Two KS-triples are conjugate under $G$ to the same sl(2)-triple in $\mathfrak{g}$ if and only if the KS-triples are conjugate under $K$.

(2) Every normal sl(2)-triple $\left\{x^{\prime}, e^{\prime}, f^{\prime}\right\}$ in $\mathfrak{g}_{\mathrm{C}}$ is conjugate under $K_{\mathrm{C}}$ to a KStriple in $\mathfrak{g}_{\mathrm{C}}$. Two KS-triples are conjugate under $K_{\mathrm{C}}$ to the same normal sl(2)-triple in $\mathfrak{g}_{\mathrm{C}}$ if and only if the KS-triples are conjugate under $K$.

Definition 2.6. Let $K S(\mathfrak{g})$ denote the set of KS-triples in $\mathfrak{g}$ and $K S\left(\mathfrak{g}_{\mathrm{C}}\right)$ denote the set of KS-triples in $\mathfrak{g}_{\mathrm{C}} \cdot K S(\mathfrak{g}) / K$ and $K S\left(\mathfrak{g}_{\mathrm{C}}\right) / K$ will denote the set of $K$ conjugacy classes in $K S(\mathfrak{g})$ and $K S\left(\mathfrak{g}_{\mathrm{C}}\right)$ respectively.

Combining Theorems 2.3, 2.2 and 2.5, we have:

Theorem 2.7. There are bijections:

$$
\mathcal{N}[\mathfrak{g}] / G \longleftrightarrow K S(\mathfrak{g}) / K
$$

and

$$
\mathcal{N}\left[\mathfrak{p}_{\mathbf{C}}\right] / K_{\mathbf{C}} \longleftrightarrow K S\left(\mathfrak{g}_{\mathrm{C}}\right) / K
$$

The Kostant-Sekiguchi correspondence is a consequence of Theorem 2.7 and the following observation.

Proposition 2.8. There is a bijection:

$$
K S(\mathfrak{g}) / K \longleftrightarrow K S\left(\mathfrak{g}_{\mathrm{C}}\right) / K
$$

defined as follows: $\{H, E, F\} \in K S(\mathfrak{g})$ is mapped to the $\{x, e, f\} \in K S\left(\mathfrak{g}_{\mathrm{C}}\right)$, where

$$
x=i(E-F), \quad e=\frac{E+F+i H}{2}, \quad f=\frac{E+F-i H}{2} .
$$

The inverse map sends $\{x, e, f\} \in K S\left(\mathfrak{g}_{\mathrm{C}}\right)$ to $\{H, E, F\} \in K S(\mathfrak{g})$, where

$$
H=-i(e-f), \quad E=\frac{e+f-i x}{2}, \quad F=\frac{e+f+i x}{2} .
$$

The maps just defined are clearly $K$-equivariant.

Definition 2.9. The triples $\{H, E, F\}$ and $\{x, e, f\}$ in equations (2.3) and (2.4) are said to be associated.

Remark 2.10. Let $\{H, E, F\} \in \operatorname{KS}(\mathfrak{g})$ and $\{x, e, f\} \in \mathrm{KS}\left(\mathfrak{g}_{\mathrm{C}}\right)$. If these triples are associated, then the nilpotents $E, F, e, f$ are in the same $G_{\mathbf{C}}$ conjugacy class in $\mathfrak{g}_{\mathrm{C}}$. 
We define a bijection $\mathcal{S}$ between $\mathcal{N}[\mathfrak{g}] / G$ and $\mathcal{N}\left[\mathfrak{p}_{\mathrm{C}}\right] / K_{\mathrm{C}}$ as follows.

Let $\Omega$ be a conjugacy class in $\mathcal{N}[\mathfrak{g}] / G$. Let $\{H, E, F\}=\left\{H_{\Omega}, E_{\Omega}, F_{\Omega}\right\}$ be a representative of the conjugacy class in $K S(\mathfrak{g})$ that is associated to $\Omega$ by Theorem [2.7. Then

$$
\mathcal{S}(\Omega) \stackrel{\text { def }}{=} K_{\mathrm{C}} \cdot \frac{E+F+i H}{2} .
$$

$\mathcal{S}^{-1}: \mathcal{N}\left[\mathfrak{p}_{\mathrm{C}}\right] / K_{\mathrm{C}} \rightarrow \mathcal{N}[\mathfrak{g}] / G$ is given as follows. Let $\mathcal{O}$ be a conjugacy class in $\mathcal{N}\left[\mathfrak{p}_{\mathbf{C}}\right] / K_{\mathrm{C}}$. Let $\{x, e, f\}=\left\{x_{\mathcal{O}}, e_{\mathcal{O}}, f_{\mathcal{O}}\right\}$ be a representative of the conjugacy class in $K S\left(\mathfrak{g}_{\mathrm{C}}\right)$ that is associated to $\mathcal{O}$ by Theorem 2.7. Then

$$
\mathcal{S}^{-1}(\mathcal{O}) \stackrel{\text { def }}{=} G \cdot \frac{-i x+e+f}{2} .
$$

We obtain our main result.

Theorem 2.11 (The Kostant-Sekiguchi Correspondence 12]). The mapping $\mathcal{S}$ : $\mathcal{N}[\mathfrak{g}] / G \longrightarrow \mathcal{N}\left[\mathfrak{p}_{\mathrm{C}}\right] / K_{\mathrm{C}}$, given by $\Omega \mapsto \mathcal{S}(\Omega) \stackrel{\text { def }}{=} \mathcal{O}_{\Omega}($ see (2.5) $)$ is a bijection whose inverse is given by (2.6).

Definition 2.12. The pair $(\Omega, \mathcal{O})$ in $\mathcal{N}[\mathfrak{g}] / G \times \mathcal{N}\left[\mathfrak{p}_{\mathrm{C}}\right] / K_{\mathrm{C}}$ is said to be a KS (Kostant-Sekiguchi) pair if $\mathcal{O}=\mathcal{S}(\Omega)$ where $\mathcal{S}$ is the Kostant-Sekiguchi bijection of Theorem 2.11. We will often write $\Omega_{\mathcal{O}}$ in place of $\mathcal{S}^{-1}(\mathcal{O})$ and $\mathcal{O}_{\Omega}$ in place of $\mathcal{S}(\Omega)$.

\section{COMPleXity AND NilPotent ORBITS}

Most of the results in this section are due to Panyushev. We require some additional notation.

Definition 3.1. Let $\mathbf{u}=\mathfrak{k}+i \mathfrak{p}$. Then $\mathbf{u}$ is a compact real form of $\mathfrak{g}_{\mathrm{C}} \cdot \tau=\sigma \circ \theta$ is the conjugation on $\mathfrak{g}_{\mathrm{C}}$ with respect to $\mathbf{u}$. Let $\mathbf{U}$ be the connected subgroup of $G_{\mathrm{C}}$ with Lie algebra $\mathbf{u}$.

Definition 3.2. Let $B$ denote the Killing form of $\mathfrak{g}_{\mathrm{C}}$ and $B_{\mathfrak{g}}$ denote the restriction of $B$ to $\mathfrak{g}$. Set $\langle z, w\rangle=-B_{\mathfrak{g}_{\mathbf{C}}}(z, \tau(w))$. Then $\langle\cdot, \cdot\rangle$ is a $\mathbf{U}$-invariant, positive definite Hermitian inner product on $\mathfrak{g}_{\mathrm{C}}$. If $z \in \mathfrak{g}_{\mathrm{C}}$, set $\|z\|^{2}=\langle z, z\rangle=-B(z, \tau(z))$. Note that if $z, w \in \mathfrak{g}$, then $\langle z, w\rangle=-B_{\mathfrak{g}}(w, \theta(w))$.

If $M$ is a real (respectively, complex) manifold, then $\operatorname{dim} M$ denotes its real (respectively, complex) dimension. If $M$ is complex, we write $\operatorname{dim}_{\mathbf{R}} M$ or $\operatorname{dim}_{\mathbf{R}} M_{\mathbf{R}}$ for the dimension of the underlying real manifold.

Our first goal is to develop some necessary and sufficient conditions for $\mathcal{O}$ to be $K_{\mathbf{C}}$ spherical. We assume that $\mathcal{O}=K_{\mathbf{C}} \cdot e$ where $e$ belongs to a KS-triple $\{x, e, f\}$. If $j$ is an integer, $\mathfrak{g}_{\mathbf{C}}(j), \mathfrak{k}_{\mathbf{C}}(j)$ and $\mathfrak{p}_{\mathbf{C}}(j)$ will denote the $j$-eigenspace of $\operatorname{ad}(x)$ in $\mathfrak{g}_{\mathrm{C}}, \mathfrak{k}_{\mathrm{C}}$ and $\mathfrak{p}_{\mathrm{C}}$ respectively. Let $\mathfrak{u}$ denote the sum of the positive eigenspaces of $\operatorname{ad}(x)$ on $\mathfrak{g}_{\mathrm{C}}$. Set

$$
Z=\mathfrak{u} \cap \mathfrak{k}_{\mathbf{C}} /\left(\mathfrak{u} \cap \mathfrak{k}_{\mathbf{C}}\right)^{e} .
$$

Definition 3.3. Let $\mathfrak{m}$ be the orthogonal complement (relative to $\langle\cdot, \cdot\rangle$ ) of $\mathfrak{k}_{\mathbf{C}}\{x, e, f\}$ inside $\mathfrak{k}_{\mathbf{C}}{ }^{x}$. 
Recall from [9] the notion of a stabilizer in general position (s.g.p.) for the action of an algebraic group on an irreducible variety, where $\mathfrak{m}$ is a $K_{\mathbf{C}}^{\{x, e, f\}}$-module. Let

$$
\mathbf{S} \text { be a s.g.p. for the action of } K_{\mathbf{C}}^{\{x, e, f\}} \text { on } \mathfrak{m} \text {. }
$$

That is, $\mathbf{S}$ is the stabilizer in $K_{\mathbf{C}}^{\{x, e, f\}}$ of a point whose orbit under $K_{\mathbf{C}}^{\{x, e, f\}}$ has maximal dimension. Such a point lies in an open subset of $\mathfrak{m}$ such that the stabilizers of any two points in this subset are conjugate under $K_{\mathbf{C}}^{\{x, e, f\}}$. Since $K_{\mathbf{C}}^{\{x, e, f\}}$ is reductive, a generic $K_{\mathbf{C}}^{\{x, e, f\}}$-orbit on $\mathfrak{m}$ is closed, so that $\mathbf{S}$ is reductive. Also,

$$
\operatorname{dim} K_{\mathbf{C}}^{\{x, e, f\}}-\operatorname{dim} \mathbf{S}=\max \operatorname{dim}_{z \in \mathfrak{m}} K_{\mathbf{C}}^{\{x, e, f\}} \cdot z .
$$

Suppose that $X$ is a variety with $K_{\mathrm{C}}$-action and that $B_{k}$ is a Borel subalgebra of $K_{\mathrm{C}}$. The complex codimension of a generic $B_{k}$-orbit is called the complexity of $X$, denoted $c_{K_{\mathbf{C}}}(X)$ or $c(X)$ (when the reductive group $K_{\mathbf{C}}$ is understood). It is also the transcendence degree (over $\mathbf{C}$ ) of the $B_{k}$ invariant functions in the field of rational functions (with complex coefficients) on $X$. Thus, $X$ is spherical for $K_{\mathrm{C}}$ if and only if $c(X)=0$. If $U_{k}$ is the nilpotent radical of $B_{k}$ and $B_{k} \cdot z$ is a generic $B_{k}$-orbit, then the codimension of $U_{k} \cdot z$ in $B_{k} \cdot z$ is called the rank of $X$. It is denoted by $r_{K_{\mathbf{C}}}(X)$ or $r(X)$. The rank of $X$ is also the transcendence degree of the $U_{k}$-invariants in the field of rational functions on $X$. Note that by results of Panyushev, when $X$ is spherical, the rank of $X$ is equal to the rank of the lattice of highest weights associated to the $B_{k}$ semi-invariant rational functions on $X$.

\section{Proposition 3.4.}

$$
c_{K_{\mathbf{C}}}\left(K_{\mathbf{C}} \cdot e\right)=c_{K_{\mathbf{C}}^{x}}\left(K_{\mathbf{C}}^{x} / K_{\mathbf{C}}\{x, e, f\}\right)+c_{S}(Z) .
$$

That is, the complexity of $K_{\mathbf{C}} \cdot e$ is the sum of the complexity of $K_{\mathbf{C}}^{x} / K_{\mathbf{C}}\{x, e, f\}$ (w.r.t. $K_{\mathbf{C}}^{x}$ ) and the complexity of $Z$ (w.r.t. $\left.\mathbf{S}\right)$.

Proof. This is Theorem 1.2(a) of [10]. (See also Theorem 2.3(a) of [10].)

Corollary 3.5. $\mathcal{O}$ is spherical if and only if $K_{\mathbf{C}}^{x} / K_{\mathbf{C}}{ }^{\{x, e, f\}}$ is spherical and a Borel subgroup of $\mathbf{S}$ has an open orbit on $Z$.

Proof. This is Corollary 1.4 of [10].

We will also require the following proposition in the proof of our main result.

Proposition 3.6. If $K_{\mathrm{C}}$ is a complex reductive algebraic group, $V$ is a finitedimensional (complex) representation of $K_{\mathrm{C}}$, and $V^{*}$ is the dual representation of $K_{\mathbf{C}}$, then

$$
\operatorname{trdeg} \mathbf{C}\left(V \times V^{*}\right)^{K_{\mathbf{C}}}=\operatorname{trdeg} \mathbf{C}(V)^{B_{k}}+\operatorname{trdeg} \mathbf{C}(V)^{U_{k}},
$$

where $\mathbf{C}\left(V \times V^{*}\right)$ denotes the field of rational functions on $V \times V^{*}=V \oplus V^{*}, K_{\mathbf{C}}$ acts on $V \times V^{*}$ via $k \cdot(v, \lambda)=(k \cdot v, k \cdot \lambda)$ (for $\left.v \in V, \lambda \in V^{*}\right)$, and "trdeg" denotes the transcendence degree over the field $\mathbf{C}$.

Proof. This follows from Corollary 1.4.1 in [9].

The previous proposition is equivalent to

$$
\operatorname{trdeg} \mathbf{C}\left(V \times V^{*}\right)^{K_{\mathbf{C}}}=2 c(V)+r(V) .
$$




\section{Compact group actions}

In this section, we collect some useful facts about compact groups acting on manifolds. Unless otherwise indicated, $K$ is an arbitrary compact, connected Lie group.

Lemma 4.1. Suppose that $K \rightarrow G L(W)$ is a representation of $K$ on a finitedimensional real vector space $W$. Let $V=W \otimes_{\mathbf{R}} \mathbf{C}=W+i W$ be a complexification of $W$, and $K_{\mathrm{C}}$ be a complexification of $K$ such that $K \subset K_{\mathrm{C}}$, and the representation of $K$ on $W$ extends to a representation of $K_{\mathrm{C}}$ on $V$. Then the maximum complex dimension of an orbit of $K_{\mathrm{C}}$ on $V$ is equal to the maximum real dimension of an orbit of $K$ on $W$.

Proof. Let $d$ denote the largest dimension of a $K_{\mathrm{C}}$ orbit in $V$. Let $U$ be the set of points $v \in V$ with $\operatorname{dim}_{\mathbf{C}} K_{\mathbf{C}} \cdot v=d$. Then $U$ is a Zariski open subset of $V$ and so must have a nonempty intersection with the real locus $W$. Suppose that $w \in U \cap W$. Then $\mathfrak{k}_{\mathbf{C}}{ }^{w}=\mathfrak{k}^{w} \oplus i \mathfrak{k}^{w}$. Thus, $\operatorname{dim}_{\mathbf{C}} \mathfrak{k}_{\mathbf{C}}{ }^{w}=\operatorname{dim}_{\mathbf{R}} \mathfrak{k}^{w}$. It follows that $\operatorname{dim}_{\mathbf{C}} K_{\mathbf{C}} \cdot w=\operatorname{dim}_{\mathbf{R}} K \cdot w=d$. It is easy to check that $d$ is the largest dimension of a $K$ orbit in $W$.

Definition 4.2. If $H$ is a complex reductive group or a compact group, rank $H$ denotes the dimension of a maximal torus of $H$.

Let $M$ be a smooth, connected manifold. We refer the reader to [5] for the notion of a Hamiltonian $K$-action on $M$ and the notion of a Poisson $K$-action on $M$.

Definition 4.3. ([3]) $M$ is said to be multiplicity-free if $C^{\infty}(M)^{K}$, the $K$-invariant smooth functions on $M$, is an abelian Lie algebra under the Poisson bracket.

Recall also the notion of a spherical $K$-space from [5].

Definition 4.4. For a symplectic manifold $M$ with Poisson $K$-action, let $\tilde{r}$ denote the (real) codimension of a maximal torus of $K^{z}$ in a maximal torus of $K$, where $K \cdot z$ is a $K$-orbit of maximal dimension in $M$. Then $\tilde{r}$ is called the rank of the action of $K$ on $M$. Note that the (real) codimension of $K \cdot z$ in $M$ is greater than or equal to $\tilde{r}$.

Definition 4.5. $M$ is said to be a spherical $K$-space of rank $\tilde{r}$ if the codimension of a $K$-orbit of maximal dimension is equal to $\tilde{r}$.

Theorem 4.6. Let $M$ be a smooth, connected manifold with Poisson $K$-action. The following are equivalent:

(1) $M$ is multiplicity free;

(2) $M$ is a spherical $K$-space.

Proof. The equivalence of (1) and (2) is part of Theorem 3 in [5].

A nilpotent $G$-orbit $\Omega$ in $\mathfrak{g}$ is a symplectic manifold under the Kostant-Kirillov symplectic form. (See Lecture 2 in [14].) $\Omega$ is then a Hamiltonian $G$-space, hence a fortiori, a Hamiltonian $K$ where $K$ is the maximal compact subgroup of $G$ defined in the introduction. The $K$-action on $\Omega$ is also Poisson.

Proposition 4.7. Let $K$ be a compact, connected group acting on a complex vector space $V$, let $\mathbf{C}\left[V_{\mathbf{R}}\right]$ denote the complex-valued polynomial functions on $V$ and let $\mathbf{C}\left(V_{\mathbf{R}}\right)$ denote the field of fractions of $\mathbf{C}\left[V_{\mathbf{R}}\right]$. (We use the notation $\mathbf{C}\left[V_{\mathbf{R}}\right]$ rather than $\mathbf{C}[V]$, because the latter notation usually refers to the holomorphic polynomials 
on $V$, i.e., the affine coordinate ring of the affine variety $V$.) Then the transcendence degree of $\mathbf{C}\left(V_{\mathbf{R}}\right)$ over $\mathbf{C}$ satisfies the equation:

$$
\operatorname{trdeg} \mathbf{C}\left(V_{\mathbf{R}}\right)^{K}=\operatorname{dim}_{\mathbf{R}} V-\max _{v \in V} \operatorname{dim} K \cdot v .
$$

Proof. See Remark 5.1 in [2].

Remark 4.8. (Notation as in the previous proposition) The representation of $K$ on $V$ extends to a representation of $K_{\mathbf{C}}$ on $V . \mathbf{C}\left[V_{\mathbf{R}}\right]$ and $\mathbf{C}\left[V \times V^{*}\right]$ (the regular functions of $\left.V \times V^{*}\right)$ are isomorphic as rings and as $K$-modules. (This is explained in the proof of Proposition 5.1 in [2.) Thus, we have

$$
\mathbf{C}\left(V_{\mathbf{R}}\right)^{K}=\mathbf{C}\left(V \times V^{*}\right)^{K}=\mathbf{C}\left(V \times V^{*}\right)^{K} \mathbf{C} .
$$

Corollary 4.9. (Notation as in Proposition (4.7) and equation (3.4)) The real codimension of the largest $K$-orbit in $V$ is equal to $2 c_{K_{\mathbf{C}}}(V)+r_{K_{\mathbf{C}}}(V)$.

Proof. This follows from the previous remark and Propositions 4.7 and 3.6 .

\section{The GeOMEtRY of NilPotent ORBIts}

The main results in this section are due to Schmid and Vilonen 11. Throughout this section, $(\Omega, \mathcal{O})$ is a Kostant-Sekiguchi pair. $\{H, E, F\}$ is a KS-triple corresponding to $\Omega$, so that $\Omega=G \cdot E .\{x, e, f\}$ is a KS-triple corresponding to $\mathcal{O}$, so that $\mathcal{O}=K_{\mathrm{C}} \cdot e .\{H, E, F\}$ and $\{x, e, f\}$ are associated in the sense of Definition 2.9

Definition 5.1. The $K$-orbit, $C(\Omega) \stackrel{\text { def }}{=} K \cdot E$ is said to be the core of $\Omega$. The $K$-orbit, $C(\mathcal{O}) \stackrel{\text { def }}{=} K \cdot e$ is said to be the core of $\mathcal{O}$.

Because of the bijections (2.1) and (2.2), the core of a nilpotent orbit in $\mathfrak{g}$ or $\mathfrak{p}_{\mathrm{C}}$ is well defined. (Definition 5.1 is equivalent to the definition of core given in [11.) Every element $E^{\prime}$ in $C(\Omega)$ is the nilpositive element of a unique KS-triple in $\mathfrak{g}$ : $\left\{\left[E^{\prime},-\theta\left(E^{\prime}\right)\right], E^{\prime},-\theta\left(E^{\prime}\right)\right\}$. Likewise every element $e^{\prime}$ in $C(\mathcal{O})$ is the nilpositive element of a unique KS-triple in $\mathfrak{g}_{\mathrm{C}}:\left\{\left[e^{\prime}, \sigma\left(e^{\prime}\right)\right], e^{\prime}, \sigma\left(e^{\prime}\right)\right\}$. Thus, we have

Remark 5.2. There is a bijection between $C(\Omega)$ and the set of KS-triples whose nilpositive elements lie in $\Omega$, and there is a bijection between $C(\mathcal{O})$ and the set of KS-triples whose nilpositive elements lie in $\mathcal{O}$.

Definition 5.3. If $\Omega$ (resp., $\mathcal{O}$ ) is a nilpotent $G$ orbit in $\mathfrak{g}$ (resp., nilpotent $K_{\mathrm{C}}$ orbit in $\mathfrak{p}_{\mathrm{C}}$ ), then $T_{C(\Omega)}^{*}(\Omega)$ (resp., $T_{C(\mathcal{O})}^{*}(\mathcal{O})$ ) denotes the conormal bundle of $C(\Omega)$ (resp., $C(\mathcal{O})$ ) inside the cotangent bundle $T^{*}(\Omega)$ (resp., $T^{*}(\mathcal{O})$ ).

Since $E \in C(\Omega)$, the tangent spaces to $\Omega$ and $C(\Omega)$ at $E$ can be described as follows: $T_{E}(\Omega) \simeq \mathfrak{g} / \mathfrak{g}^{E} \simeq[\mathfrak{g}, E]$ and $T_{E}(C(\Omega)) \simeq \mathfrak{k} / \mathfrak{k}^{E} \simeq[\mathfrak{k}, E]$. Thus the fiber of $T_{C(\Omega)}^{*}(\Omega)$ at $E$ is given by

$$
T_{C(\Omega)}^{*}(\Omega)(E) \simeq[\mathfrak{g}, E] /[\mathfrak{k}, E] .
$$

Note that the fiber $T_{C(\Omega)}^{*}(\Omega)(E)$ is a $K^{E}$-module. Likewise, since $e \in C(\mathcal{O})$,

$$
T_{C(\mathcal{O})}^{*}(\mathcal{O})(e) \simeq\left[\mathfrak{k}_{\mathbf{C}}, e\right] /[\mathfrak{k}, e] .
$$

Schmid and Vilonen discovered that there is a close relationship between $\Omega$ and $T_{C(\Omega)}^{*}(\Omega)$. 
Proposition 5.4 ([11). Let $\Omega=G \cdot E$ be a nilpotent orbit in $\mathfrak{g}$, and suppose $C(\Omega)=K \cdot E$. Then, there is a $K$-equivariant diffeomorphism mapping $\Omega$ onto $T_{C(\Omega)}^{*}(\Omega)$.

Likewise, there is a similar relationship between $\mathcal{O}$ and $T_{C(\mathcal{O})}^{*}(\mathcal{O})$.

Proposition 5.5 (Schmid-Vilonen). There is a K-equivariant diffeomorphism mapping $\mathcal{O}$ onto $T_{C(\mathcal{O})}^{*}(\mathcal{O})$.

Proof. We follow the proof of Proposition [5.4 in [11].

We need the following additional notation:

$$
\begin{aligned}
\mathfrak{s} & =\text { the real span of the triple }\{H, E, F\}, \\
\mathfrak{l} & =\mathfrak{k}_{\mathrm{C}}^{x} \\
\mathfrak{u} & =\text { the direct sum of the positive eigenspaces of } \operatorname{ad}(x) \text { on } \mathfrak{k}_{\mathrm{C}}, \\
\mathfrak{q} & =\mathfrak{l} \oplus \mathfrak{u}, \\
L & =K_{\mathrm{C}}^{x} \\
U & =\exp (\mathfrak{u}), \\
\mathfrak{u}_{e} & =\mathfrak{u} \cap \mathfrak{k}_{\mathrm{C}}{ }^{e} .
\end{aligned}
$$

Choose $\mathfrak{r}$ to be the orthogonal complement relative to $\langle\cdot, \cdot\rangle$ (Definition 3.2) of $\mathfrak{u}_{e}$ inside $\mathfrak{u}$. Then $\mathfrak{r}$ is invariant under $K^{e}$ and $L^{e}$.

Definition 5.6. With notation as above, set

$$
V_{\mathcal{O}}(\mathfrak{s})=\left(i \mathfrak{k}^{x} \cap\left(i \mathfrak{k}^{\mathfrak{s}}\right)^{\perp}\right) \oplus \mathfrak{r} .
$$

There are two key facts that must be established to prove the proposition:

(1) there is a $K$-equivariant diffeomorphism

$$
\mathcal{O} \longrightarrow K \times_{K^{\mathfrak{s}}} V_{\mathcal{O}}(\mathfrak{s}),
$$

and

(2) there is a $K^{\mathfrak{s}}$-module isomorphism

$$
V_{\mathcal{O}}(\mathfrak{s}) \simeq\left[\mathfrak{k}_{\mathbf{C}}, e\right] /[\mathfrak{k}, e] .
$$

(The right-hand side of (5.3) is the fiber of $T_{C(\mathcal{O})}^{*}(\mathcal{O})$ at e.)

Recall that $Q=L U$ is a parabolic subgroup of $K_{\mathrm{C}}$. Moreover, $K_{\mathrm{C}}^{e}=L^{e} U^{e}=Q^{e}$. By hypothesis, $e=\sigma(f)$, so that $\mathfrak{k}^{e}=\mathfrak{k}^{f}$ and $K^{e}=K^{f}$. Since any two elements of an sl(2)-triple determine the third, and $\{H, E, F\}$ and $\{x, e, f\}$ are related by (2.3), we have $l^{e}=\mathfrak{k}_{\mathrm{C}}^{\mathfrak{s}}$ and $K^{e}=K^{\mathfrak{s}}$.

Since $K_{\mathrm{C}}=K Q$, we can write $K_{\mathrm{C}}$ as the fiber product:

$$
K_{\mathrm{C}} \simeq K \times_{K \cap L}(L U)=K \times_{K^{x}}(L U) .
$$

Since $\mathfrak{r}$ is invariant under $K^{e}$ and $L^{e}$, the diffeomorphism

$$
\mathfrak{r} \times \mathfrak{u}_{e} \rightarrow U, \quad(X, Y) \mapsto \exp (X) \exp (Y)
$$

is equivariant for $K^{e}$ and $L^{e}$. Starting with (5.4) and taking advantage of (5.5), we obtain

$$
\begin{aligned}
K_{\mathrm{C}} & \simeq K \times_{K^{x}}\left(L \times_{L^{\mathfrak{s}}} L^{\mathfrak{s}} U\right) \\
& \simeq K \times_{K^{x}}\left(L \times_{L^{\mathfrak{s}}}\left(L^{\mathfrak{s}} \times \mathfrak{r} \times U^{e}\right)\right),
\end{aligned}
$$


where $L^{\mathfrak{s}}$ acts on the direct product $L^{\mathfrak{s}} \times \mathfrak{r} \times U^{e}$ by left translation on the first factor, the adjoint action on the second factor and conjugation on the last factor. We can rearrange the factors in the last fiber product in (5.6) to obtain

$$
\begin{aligned}
K_{\mathrm{C}} & \simeq K \times_{K^{x}}(L \times \mathfrak{r}) \times_{L^{\mathfrak{s}}}\left(L^{\mathfrak{s}} \times U^{e}\right) \\
& \simeq K \times_{K^{x}}(L \times \mathfrak{r}) \times_{L^{\mathfrak{s}}} K_{\mathrm{C}}^{e} \quad\left(\text { since } K_{\mathrm{C}}^{e}=L^{e} U^{e}\right) .
\end{aligned}
$$

$\tau$ (Definition 3.1) is a Cartan involution of $\mathfrak{l}$. So $\mathfrak{l}$ has Cartan decomposition $\mathfrak{k}^{x} \oplus i \mathfrak{k}^{x}$. The orthogonal complement of $i \mathfrak{k}^{\mathfrak{s}}$ (relative to the inner product $\langle\cdot, \cdot\rangle$ ) inside $i \mathfrak{k}^{x}$ is equal to $i \mathfrak{k}^{x} \cap\left(i \mathfrak{k}^{\mathfrak{s}}\right)^{\perp}$, where $\left(i \mathfrak{k}^{\mathfrak{s}}\right)^{\perp}$ is the orthogonal complement of $i \mathfrak{k}^{\mathfrak{s}}$ in $\mathfrak{k}_{\mathrm{C}}$. This fact allows us to refine the usual diffeomorphism: $L=K^{x} \exp \left(i \mathfrak{k}^{x}\right)$. It follows from Theorem 5 of [8] that

$$
L=K^{x} \exp \left(i \mathfrak{k}^{x} \cap\left(i \mathfrak{k}^{\mathfrak{s}}\right)^{\perp}\right) \exp \left(i \mathfrak{k}^{\mathfrak{s}}\right),
$$

where the left-hand side is a topological product. We now have

$$
\begin{aligned}
L & \simeq\left(K^{x}\right) \times_{K^{\mathfrak{s}}} K^{\mathfrak{s}} \exp \left(i \mathfrak{k}^{x} \cap\left(i \mathfrak{k}^{\mathfrak{s}}\right)^{\perp}\right) \exp \left(i \mathfrak{k}^{\mathfrak{s}}\right) \\
& \simeq\left(K^{x}\right) \times_{K^{\mathfrak{s}}} \exp \left(i \mathfrak{k}^{x} \cap\left(i \mathfrak{k}^{\mathfrak{s}}\right)^{\perp}\right) K^{\mathfrak{s}} \exp \left(i \mathfrak{k}^{\mathfrak{s}}\right) \\
& \simeq\left(K^{x}\right) \times_{K^{\mathfrak{s}}} \exp \left(i \mathfrak{k}^{x} \cap\left(i \mathfrak{k}^{\mathfrak{s}}\right)^{\perp}\right) L^{\mathfrak{s}} .
\end{aligned}
$$

Now substitute the final expression for $L$ in (5.8) into the decomposition for $K_{\mathrm{C}}$ in (5.7) and rearrange some of the factors to obtain

$$
\begin{aligned}
K_{\mathrm{C}} & \simeq K \times_{K^{x}}\left(\left(K^{x}\right) \times_{K^{\mathfrak{s}}} \exp \left(i \mathfrak{k}^{x} \cap\left(i \mathfrak{k}^{\mathfrak{s}}\right)^{\perp}\right) \times \mathfrak{r} \times L^{\mathfrak{s}} \times_{L^{\mathfrak{s}}} L^{\mathfrak{s}}\right. \\
& \simeq K \times_{K^{\mathfrak{s}}} \exp \left(i \mathfrak{k}^{x} \cap\left(i \mathfrak{k}^{\mathfrak{s}}\right)^{\perp}\right) \times \mathfrak{r} \times K_{\mathrm{C}}^{e} .
\end{aligned}
$$

Since $\mathcal{O}=K_{\mathrm{C}} / K_{\mathrm{C}}^{e}$, this argument shows that there is a $K$-equivariant diffeomorphism

$$
K \times_{K^{\mathfrak{s}}}\left(\left(i \mathfrak{k}^{x} \cap\left(i \mathfrak{k}^{\mathfrak{s}}\right)^{\perp}\right) \oplus \mathfrak{r}\right) \longrightarrow \mathcal{O}
$$

$$
\text { given by }[k, S+T] \mapsto k \exp (S) \exp (T) \cdot e \text {. }
$$

This diffeomorphism is also $\mathbf{R}^{+}$-equivariant for the obvious $\mathbf{R}^{+}$-action on $\mathcal{O}$ and the action

$$
t \diamond[k, S+T]=\left[k,\left(\left(\frac{\ln (t)}{2} x+S\right)+T\right]\right.
$$

on $K \times_{K^{\mathfrak{s}}}\left(\left(i \mathfrak{k}^{x} \cap\left(i \mathfrak{k}^{\mathfrak{s}}\right)^{\perp}\right) \oplus \mathfrak{r}\right)$. It remains to show that $\left(\left(i \mathfrak{k}^{x} \cap\left(i \mathfrak{k}^{\mathfrak{s}}\right)^{\perp}\right) \oplus \mathfrak{r}\right)$ is isomorphic as a $K^{\mathfrak{s}}$-module to $\left[\mathfrak{k}_{\mathrm{C}}, e\right] /[\mathfrak{k}, e]$, the fiber of $T_{C(\mathcal{O})}^{*}(\mathcal{O})$ at $e$. To do this, we establish a subspace decomposition of $\left[\mathfrak{k}_{\mathrm{C}}, e\right]$ analogous to the decomposition of $[\mathfrak{g}, E]$ used by Schmid and Vilonen.

Let $I d$ denote the identity map on $\mathfrak{g}_{\mathrm{C}}$. Since $\sigma$ is conjugation relative to the real form $\mathfrak{g}$ of $\mathfrak{g}_{\mathrm{C}}, \sigma_{1}=\frac{I d+\sigma}{2}$ and $\sigma_{2}=\frac{I d-\sigma}{2}$ are the projections onto $\mathfrak{g}$ and $i \mathfrak{g}$ respectively. We start from the decompositions:

$$
\begin{aligned}
\mathfrak{k}_{\mathbf{C}} & =\mathfrak{k}_{\mathbf{C}}^{x} \oplus \mathfrak{u} \oplus \sigma \mathfrak{u}, \\
\mathfrak{k} & =\mathfrak{k}^{x} \oplus \sigma_{1}(\mathfrak{u}), \\
\mathfrak{k}^{x} & =\mathfrak{k}^{\mathfrak{s}} \oplus\left(\mathfrak{k}^{x} \cap\left(\mathfrak{k}^{\mathfrak{s}}\right)^{\perp}\right) .
\end{aligned}
$$

(The subspaces in these decompositions are mutually orthogonal with respect to the inner product $\langle\cdot, \cdot\rangle$.) 
We have the vector space decompositions

$$
\begin{aligned}
{\left[\mathfrak{k}_{\mathbf{C}}, e\right] } & =\left[\mathfrak{k}_{\mathbf{C}}^{x}, e\right]+[\mathfrak{u}, e]+[\sigma(\mathfrak{u}), e] \\
& =\left[\mathfrak{k}_{\mathbf{C}}^{x}, e\right]+\left[\mathfrak{r}+\mathfrak{u}_{e}, e\right]+\left[\sigma\left(\mathfrak{r}+\mathfrak{u}_{e}\right), e\right] \\
& =\left[\mathfrak{k}_{\mathbf{C}}^{x}, e\right]+[\mathfrak{r}, e]+[\sigma(\mathfrak{r}), e]+\left[\sigma\left(\mathfrak{u}_{e}\right), e\right] .
\end{aligned}
$$

Since $[\mathfrak{r}, e]$ and $[\sigma(\mathfrak{r}), e]$ are both subspaces of $\left[\sigma_{1}(\mathfrak{r}), e\right]+\left[\sigma_{2}(\mathfrak{r}), e\right]$, and $\left[\sigma_{1}\left(\mathfrak{u}_{e}\right), e\right]=$ $\left[\sigma\left(\mathfrak{u}_{e}\right), e\right]$, we have

$$
\left[\mathfrak{k}_{\mathbf{C}}, e\right]=\left[\mathfrak{k}_{\mathbf{C}}^{x}, e\right]+\left[\sigma_{1}(\mathfrak{r}), e\right]+\left[\sigma_{2}(\mathfrak{r}), e\right]+\left[\sigma_{1}\left(\mathfrak{u}_{e}\right), e\right] .
$$

Similarly, we have

$$
\begin{aligned}
{[\mathfrak{k}, e] } & =\left[\mathfrak{k}^{x}, e\right]+\left[\sigma_{1}(\mathfrak{r}), e\right]+\left[\sigma_{1}\left(\mathfrak{u}_{e}\right), e\right] \\
& =\left[\mathfrak{k}^{\mathfrak{s}} \cap\left(\mathfrak{k}^{\mathfrak{s}}\right)^{\perp}, e\right]+\left[\sigma_{1}(\mathfrak{r}), e\right]+\left[\sigma_{1}\left(\mathfrak{u}_{e}\right), e\right] .
\end{aligned}
$$

It follows that $\left[\mathfrak{k}_{\mathrm{C}}, e\right] /[\mathfrak{k}, e] \simeq\left[i\left(\mathfrak{k}^{\mathfrak{s}} \cap\left(\mathfrak{k}^{\mathfrak{s}}\right)^{\perp}\right), e\right]+\left[\sigma_{2}(\mathfrak{r}), e\right]$ as $K^{\mathfrak{s}}$-modules. The $\operatorname{sum}\left[i\left(\mathfrak{k}^{\mathfrak{s}} \cap\left(\mathfrak{k}^{\mathfrak{s}}\right)^{\perp}\right), e\right]+\left[\sigma_{2}(\mathfrak{r}), e\right]$ is direct and isomorphic to $V_{\mathcal{O}}(\mathfrak{s})$ as a $K^{\mathfrak{s}}$-module. This establishes (5.3), completing the proof of the proposition.

Remark 5.7. With notation as in Definitions [5.6, 3.3. and lines (3.1) and (3.2) above,

$$
\begin{aligned}
& \operatorname{dim}_{\mathbf{R}} i \mathfrak{k}^{x} \cap\left(i \mathfrak{k}^{\mathfrak{s}}\right)^{\perp}=\operatorname{dim}_{\mathbf{C}} \mathfrak{m}, \\
& \mathfrak{r} \simeq Z \text { as } K^{\mathfrak{s}} \text {-modules. }
\end{aligned}
$$

\section{Proof of the main Result}

Our proof depends on an important theorem of Vergne [13]:

Theorem 6.1. If $(\Omega, \mathcal{O})$ is a Kostant-Sekiguchi pair, there is a K-equivariant diffeomorphism $\mathcal{V}_{\Omega}: \Omega \rightarrow \mathcal{O}$.

Since $\{x, e, f\}$ is a KS-triple, $K_{\mathrm{C}}^{\{x, e, f\}}$ is stable under $\sigma$ and $\theta$. The same holds for the subgroup $\mathbf{S}$ defined in (3.2). Therefore, there is a compact real form $\mathbf{S}_{\mathbf{R}}$ of $\mathbf{S}$ that is contained in $K^{\mathfrak{s}}$.

Lemma 6.2. Let $d_{0}, d_{1}$, and $d_{2}$ denote the maximum (real) dimension of a $K^{\mathfrak{s}}$ orbit in the subspaces $V_{\mathcal{O}}(\mathfrak{s}), i \mathfrak{k}^{x} \cap i\left(\mathfrak{k}^{\mathfrak{s}}\right)^{\perp}$, and $\mathfrak{r}$ respectively. Then

(1) $d_{0}=d_{1}+d_{2}$,

(2) $d_{2}=\max _{z \in \mathfrak{r}} \operatorname{dim} \mathbf{S}_{\mathbf{R}} \cdot z$,

(3) $\max _{e^{\prime} \in \mathcal{O}} \operatorname{dim} K \cdot e^{\prime}=\operatorname{dim} K-\operatorname{dim} K^{\mathfrak{s}}+d_{0}$.

Proof. (1) follows from the fact that we have a $K^{\mathfrak{s}}$-module decomposition: $V_{\mathcal{O}}(\mathfrak{s})=$ $i \mathfrak{k}^{x} \cap i\left(\mathfrak{k}^{\mathfrak{s}}\right)^{\perp} \oplus \mathfrak{r}$. (3) is also clear.

Now suppose that $y \in i \mathfrak{k}^{x} \cap i\left(\mathfrak{k}^{\mathfrak{s}}\right)^{\perp}, z \in \mathfrak{r}$ and $\operatorname{dim} K^{\mathfrak{s}} \cdot(y+z)=d_{0}$. Then we must have $\operatorname{dim} K^{\mathfrak{s}} \cdot y=d_{1}$ and $\operatorname{dim} K^{\mathfrak{s}} \cdot z=d_{2}$. So we may assume that $\left(K^{\mathfrak{s}}\right)^{y}=\mathbf{S}_{\mathbf{R}}$. But then $\left(K^{\mathfrak{s}}\right)^{y+z}=\mathbf{S}_{\mathbf{R}}^{z}$. If we rewrite the equality $d_{0}=d_{1}+d_{2}$ as

$$
\operatorname{dim} K^{\mathfrak{s}}-\operatorname{dim}\left(K^{\mathfrak{s}}\right)^{y+z}=\operatorname{dim} K^{\mathfrak{s}}-\operatorname{dim}\left(K^{\mathfrak{s}}\right)^{y}+\operatorname{dim} K^{\mathfrak{s}}-\operatorname{dim}\left(K^{\mathfrak{s}}\right)^{z},
$$

that is,

$$
\operatorname{dim} K^{\mathfrak{s}}-\operatorname{dim} \mathbf{S}_{\mathbf{R}}^{z}=\operatorname{dim} K^{\mathfrak{s}}-\operatorname{dim} \mathbf{S}_{\mathbf{R}}+\operatorname{dim} K^{\mathfrak{s}}-\operatorname{dim}\left(K^{\mathfrak{s}}\right)^{z},
$$

we conclude that

$$
\operatorname{dim} \mathbf{S}_{\mathbf{R}}-\operatorname{dim} \mathbf{S}_{\mathbf{R}}^{z}=\operatorname{dim} K^{\mathfrak{s}}-\operatorname{dim}\left(K^{\mathfrak{s}}\right)^{z} .
$$

This last equation establishes (2) of the lemma. 
Remark 6.3. Assume the notation of the proof of Lemma 6.2. If 1 denotes the identity in $K$, let $\hat{e}$ denote the equivalence class $[\mathbf{1}, y+z]$ in $K \times_{K^{\mathfrak{s}}} V_{\mathcal{O}}(\mathfrak{s})$. One can check that $\operatorname{dim} K \cdot \hat{e}=d_{0}+\operatorname{dim} K-\operatorname{dim} K^{\mathfrak{s}}$ and $K^{\hat{e}}=\mathbf{S}_{\mathbf{R}}^{z}$.

Theorem 6.4. If $(\Omega, \mathcal{O})$ is a Kostant-Sekiguchi pair, the following are equivalent:

(1) $\mathcal{O}=K_{\mathbf{C}} \cdot e$ is spherical as a $K_{\mathbf{C}}$-variety;

(2) $\Omega$ is multiplicity free as a K-Hamiltonian space.

Proof. By Theorem 4.6, establishing that $\Omega$ is multiplicity free as a $K$-Hamiltonian space depends only on the $K$-orbit structure of $\Omega$. By Theorem 6.1 , we can study the $K$-orbit structure of $\mathcal{O}$ instead.

We start with a formula for the dimension of $\mathcal{O}$ :

$$
\operatorname{dim}_{\mathbf{R}} \mathcal{O}=\operatorname{dim} K-\operatorname{dim} K^{\mathfrak{s}}+\operatorname{dim}_{\mathbf{C}} \mathfrak{m}+\operatorname{dim} \mathfrak{r} .
$$

This follows from (5.2) and the fact that $\operatorname{dim}_{\mathbf{C}} \mathfrak{m}=\operatorname{dim}_{\mathbf{R}} i \mathfrak{k}^{x} \cap i\left(\mathfrak{k}^{\mathfrak{s}}\right)^{\perp}(\operatorname{Remark}[5.7)$.

From Lemma 6.2, the maximum dimension of a $K$-orbit in $\mathcal{O}$ is given by

$$
\max \operatorname{dim}_{z \in \mathcal{O}} K \cdot z=\operatorname{dim} K-\operatorname{dim} K^{\mathfrak{s}}+\operatorname{dim}_{\mathbf{C}} K_{\mathbf{C}}^{\mathfrak{s}}-\operatorname{dim}_{\mathbf{C}} \mathbf{S}+d_{2} .
$$

It follows from (6.3) and (6.4) that the codimension of a $K$-orbit of maximum dimension is

$$
\operatorname{dim}_{\mathbf{C}} \mathfrak{m}-\left(\operatorname{dim}_{\mathbf{C}} K_{\mathbf{C}}^{\mathfrak{s}}-\operatorname{dim}_{\mathbf{C}} \mathbf{S}\right)+\operatorname{dim} \mathfrak{r}-d_{2}
$$

By Theorem 5 in 9 ,

$$
\begin{aligned}
\operatorname{dim}_{\mathbf{C}} \mathfrak{m}-\left(\operatorname{dim}_{\mathbf{C}} K_{\mathbf{C}}^{\mathfrak{s}}-\operatorname{dim}_{\mathbf{C}} \mathbf{S}\right) & =\operatorname{rank} K_{\mathbf{C}}^{x}-\operatorname{rank} \mathbf{S}+2 c_{K_{\mathbf{C}}^{x}}\left(\mathfrak{k}_{\mathbf{C}}^{x} / K_{\mathbf{C}}^{\mathfrak{s}}\right) \\
& =\operatorname{rank} K-\operatorname{rank} \mathbf{S}_{\mathbf{R}}+2 c_{K_{\mathbf{C}}^{x}}\left(K_{\mathbf{C}}^{x} / K_{\mathbf{C}}^{\mathfrak{s}}\right) .
\end{aligned}
$$

By Lemma $6.2 \operatorname{dim} \mathfrak{r}-d_{2}$, the codimension of a generic $K^{\mathfrak{s}}$-orbit in $\mathfrak{r}$, equals the codimension of a generic $\mathbf{S}_{\mathbf{R}}$ orbit in $\mathfrak{r}$. Since $\mathfrak{r}$ and $Z$ are isomorphic as $K^{\mathfrak{s}}$-modules, and hence as $\mathbf{S}_{\mathbf{R}}$-modules, Proposition 4.7 and Corollary 4.9 imply that:

$$
\operatorname{dim} \mathfrak{r}-d_{2}=\operatorname{trdeg} \mathbf{C}[\mathfrak{r}]^{\mathbf{S}_{\mathbf{R}}}=\operatorname{trdeg} \mathbf{C}\left[Z \times Z^{*}\right]^{\mathbf{S}}=2 c_{\mathbf{S}}(Z)+r_{\mathbf{S}}(Z) .
$$

We now combine ([6.5), (6.6), (6.7), and the fact that $r_{\mathbf{S}}(Z)=\operatorname{rank} \mathbf{S}_{\mathbf{R}}-\operatorname{rank} \mathbf{S}_{\mathbf{R}}{ }^{z}$ $(z$ as in (6.1) ) to conclude that the codimension of a $K$-orbit in $\mathcal{O}$ of maximal dimension is given by

$$
\begin{aligned}
& \operatorname{rank} K-\operatorname{rank} \mathbf{S}_{\mathbf{R}}+2 c_{K_{\mathbf{C}}^{x}}\left(K_{\mathbf{C}}^{x} / K_{\mathbf{C}}^{\mathfrak{s}}\right)+2 c_{\mathbf{S}}(Z)+\operatorname{rank} \mathbf{S}_{\mathbf{R}}-\operatorname{rank} \mathbf{S}_{\mathbf{R}}{ }^{z} \\
& =\operatorname{rank} K-\operatorname{rank} \mathbf{S}_{\mathbf{R}}^{z}+2 c_{K_{\mathbf{C}}^{x}}\left(K_{\mathbf{C}}^{x} / K_{\mathbf{C}}^{\mathfrak{s}}\right)+2 c_{\mathbf{S}}(Z) .
\end{aligned}
$$

We now complete the proof of the theorem:

$(1) \Longrightarrow(2)$. If $\mathcal{O}$ is spherical, then Corollary 3.5 and equation (6.8) imply that $c_{K_{\mathbf{C}}^{x}}\left(K_{\mathbf{C}}^{x} / K_{\mathbf{C}}^{\mathfrak{s}}\right)=0$ and $c_{\mathbf{S}}(Z)=0$. Thus, the codimension of a $K$-orbit of maximal dimension in $\mathcal{O}$ and hence in $\Omega$ is equal to $\operatorname{rank} K-\operatorname{rank} \mathbf{S}_{\mathbf{R}}{ }^{z}$. (See Remark 6.3) So by Theorem 4.6 , $\Omega$ is multiplicity free.

$(2) \Longrightarrow(1)$. If $\Omega$ is multiplicity free, the codimension of a $K$-orbit of maximal dimension in $\mathcal{O}$ is equal to $\operatorname{rank} K-\operatorname{rank} \mathbf{S}_{\mathbf{R}}{ }^{z}$. So equation (6.8) implies that $c_{K_{\mathbf{C}}^{x}}\left(K_{\mathbf{C}}^{x} / K_{\mathbf{C}}^{\mathfrak{s}}\right)=0$ and $c_{\mathbf{S}}(Z)=0$. By Corollary 3.5 , one concludes that $\mathcal{O}$ is spherical.

Corollary 6.5. Suppose $\mathfrak{g}$ is a complex semisimple Lie algebra and $\mathcal{O}$ is a nilpotent $G$-orbit. Then $\mathcal{O}$ is spherical for $G$ if and only if $\mathcal{O}$ is multiplicity-free as a Hamiltonian $K$-space. 
Corollary 6.5 is an immediate consequence of Theorem 6.4 because the KostantSekiguchi correspondence maps $\mathcal{O}$ to itself. The corollary provides us with many examples of noncompact multiplicity-free spaces because the spherical nilpotent orbits of complex semisimple Lie algebras have been classified by Panyushev and McGovern.

\section{REFERENCES}

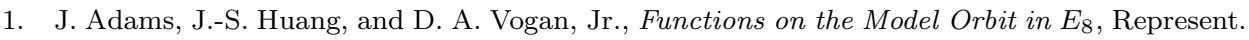
Theory, 2(1998), 224-263. MR 99g:20077

2. C. Benson, J. Jenkins, R. L. Lipsman, and G. Ratcliff, A Geometric Criterion for Gelfand Pairs Associated with the Heisenberg Group, Pacific J. Math., 178(1)(1997), 1-36. MR 98i:22012

3. Victor Guillemin and Shlomo Sternberg, Multiplicity-free spaces, J. Differential Geometry 19(1984), 31-56. MR 85h:58071

4. Victor Guillemin and Shlomo Sternberg, Symplectic techniques in physics, Cambridge University Press, Cambridge, 1984. MR 86f:58054

5. A. T. Huckleberry and T. Wurzbacher, Multiplicity-free complex manifolds, Math. Ann., 286(1990), 261-280. MR 91c:32027

6. B. Kostant and S. Rallis, Orbits and representations associated with symmetric spaces, Amer. J. Math, 93(1971), 753-809. MR 47:399

7. W. McGovern, Rings of regular functions on nilpotent orbits II: Model Algebras and Orbits, Comm. Algebra, 22(3)(1994), 765-772. MR 95b:22035

8. G. D. Mostow, Some new decomposition theorems for semi-simple groups, Amer. Math. Soc. Memoirs, 14(1955), 31-54. MR 16:1087g

9. Dmitri I. Panyushev, Complexity and rank of homogeneous spaces, Geometriae Dedicata 34 (1990), 249-269. MR 92e:14046

10. Dmitri I. Panyushev, Complexity and nilpotent orbits, Manuscripta Math. 83 (1994), 223-237. MR 95e:14039

11. Wilfrid Schmid and Kari Vilonen, On the geometry of nilpotent orbits. Sir Michael Atiyah: a great mathematician of the twentieth century, Asian J. Math., 3(1999)(1), 233-274. MR 2000g:22021

12. J. Sekiguchi, Remarks on real nilpotent orbits of a symmetric pair, J. Math. Soc. Japan, 39(1), (1987), 127-138. MR 88g:53053

13. Michèle Vergne, Instantons et correspondance de Kostant-Sekiguchi, C. R. Acad. Sci. Paris 320(1995), Série 1, 901-906. MR 96c:22026

14. David A. Vogan Jr., The method of coadjoint orbits for real reductive groups, IAS/Park City Mathematics Series, 8, Amer. Math. Soc., Providence, RI, 2000. MR 2001k:22027

15. David A. Vogan Jr., Singular unitary representations, 506-535, in Noncommutative Harmonic Analysis and Lie Groups, J. Carmona and M. Vergne, eds., Lecture Notes in Mathematics 880, Springer-Verlag, Berlin-Heidelberg-New York, 1981. MR 83k:22036

Department of Mathematics, Northeastern University, Boston, Massachusetts 02115

E-mail address: donking@neu.edu 